\section{La voie cGAS-STING}

\section{Une nouvelle arme « innée » contre le cancer}

Hisoilat Bacar ${ }^{1}$, Marie-Cécile Michallet ${ }^{2}$
${ }^{1}$ Master 2 biologie du cancer, ISPB Université Claude Bernard Lyon 1, France. ${ }^{2}$ TERI (Tumor Escape, Resistance and Immunity), Centre de recherche en cancérologie de Lyon, Centre Léon Bérard, Université de Lyon, Université Claude Bernard Lyon 1, Inserm 1052, CNRS 5286, 69008 Lyon, France. hisoilat.bacar@etu.univ-lyonl.fr

Marie-cecile.michallet@lyon.unicancer.fr

possible protection anti-tumorale, aux étapes précoces de la tumorigenèse. À la suite d'irradiations, la formation de micronoyaux, conséquence d'une instabilité chromosomique induite par des dommages à l'ADN, a ainsi pu être observée dans les cellules au cours de la mitose $[3,4]$. La rupture de l'enveloppe de ces structures entraîne une accumulation de cGAS. cGAS est alors activé au contact de la chromatine présente dans les micronoyaux, induisant ainsi la production d'IFN-I, puis l'expression des ISG (interferon-stimulated genes) [3]. Des fragments de chromatine cytosolique engendrés dans différentes conditions de sénescence (réplicative, liée à des dommages à l'ADN, ou induite par la surexpression d'un oncogène) ont également été décrits comme activant la voie cGAS-STING, et comme étant à l'origine de la production des cytokines du SASP (senescent-associated secretory phenotype), un ensemble de cytokines produites par les cellules sénescentes [5]. L'IFN-I a un rôle clé dans la réponse antitumorale. Il permet l'induction d'une réponse des lymphocytes $\mathrm{TCD}^{+}$cytotoxiques contre les antigènes tumoraux [6]. Cette réponse dépend de l'activation des cellules dendritiques (DC) qui expriment à leur surface le récepteur de I'IFN-I (IFNAR). Chez la souris, la liaison de I'IFN-I à son récepteur augmente ainsi les capacités de présentation croisée des antigènes tumoraux par les DC CD $8 \alpha^{+}$, une sous-population de cellules dendritiques exprimant $\operatorname{CD} 8 \alpha[6,7]$.

L'équipe de Deng a montré que STING était nécessaire à l'induction d'IFN-I par les $D C$ en réponse à des cellules tumo- 


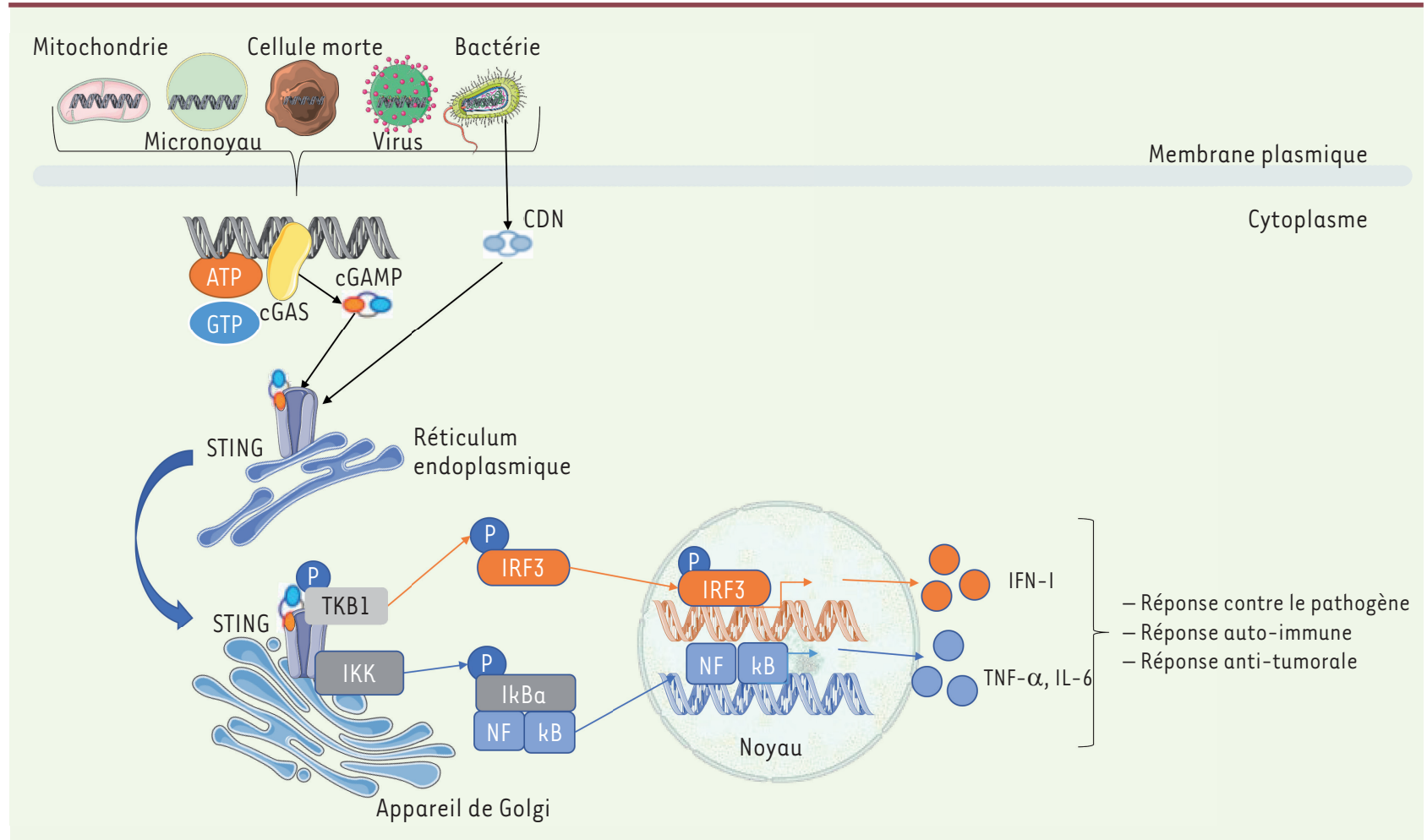

Figure 1. La voie de signalisation cGAS-STING. La synthase de GMP-AMP cyclique, cGAS, est activée après la détection d'un ADN double brin présent dans le cytosol. Cet ADN peut être d'origine bactérienne ou virale, ou d'origine endogène, provenant de cellules mortes, de la mitochondrie ou de micronoyaux. La liaison à l'ADN active cGAS qui engendre, à partir d'ATP et de GTP, des dinucléotides cycliques (CDN), comme le cGAMP, qui constituent les ligands de la protéine STING (stimulator of interferon genes) présente à la surface du réticulum endoplasmique (RE). Certaines bactéries peuvent activer directement STING via la génération de CDN, indépendamment de cGAS. La fixation de CDN à STING permet son changement de conformation et sa translocation vers l'appareil de Golgi, où il recrute la kinase TKBl qui va phosphoryler le facteur de transcription IRF3 et induire la production d'IFN-I. STING recrute également la kinase IKK nécessaire à l'activation de NFkB et à l'expression de cytokines pro-inflammatoires telles que le TNF et I'IL-6.

rales irradiées, ainsi qu'à la mise en place d'une réponse immunitaire adaptative anti-tumorale [8]. Ces observations ont ainsi révélé un rôle majeur de la protéine STING dans les cellules immunitaires. Des expériences récentes, réalisées chez la souris (dans des modèles de tumeurs transplantées), ont montré que l'expression de cGAS dans les cellules tumorales était critique pour l'activation de STING dans les cellules non tumorales (les cellules myéloïdes et les lymphocytes B). cGAMP est en effet transféré de la cellule tumorale à la cellule non tumorale (un transfert effectué possiblement via des jonctions serrées, ou par fusion entre membranes cellulaires). Ce processus aboutit au rejet de la tumeur par les cellules natural killer
(NK) qui sont indirectement activées par I'IFN-I produit par les cellules myéloïdes [9] Figure 2).

Les observations que nous avons évoquées montrent l'importance de la voie cGAS-STING dans le contrôle de la progression tumorale, en particulier aux étapes précoces de la tumorigenèse. Les cellules cancéreuses vont cependant mettre en place des mécanismes d'échappement à des stades plus tardifs, ciblant cette voie de signalisation [10]. Les analyses de bases de données, telles que cBioPortal (qui permet de visualiser, d'analyser et de télécharger des ensembles de données génomiques à grande échelle sur le cancer - www.cbioportal.org) ou COSMIC (ressource la plus importante et la plus complète au monde pour explorer l'impact des mutations somatiques dans les cancers humains - https://cancer.sanger.ac.uk/cosmic), ont en effet permis de mettre en évidence l'inhibition de la voie cGAS-STING dans une grande variété de tumeurs, celle-ci reposant sur des mutations perte de fonction ou sur des modifications épigénétiques des régions promotrices des gènes cgas et sting [11]. Une étude a par ailleurs démontré que le récepteur tyrosine kinase HER2 (également connu sous le nom de ErbB-2 ou Neu), dont l'expression est augmentée dans les cancers du sein, était un inhibiteur naturel de la voie cGAS-STING. L'effet inhibiteur de ce récepteur est critique pour empêcher l'entrée en sénescence ou en apoptose des cellules tumorales [12]. 


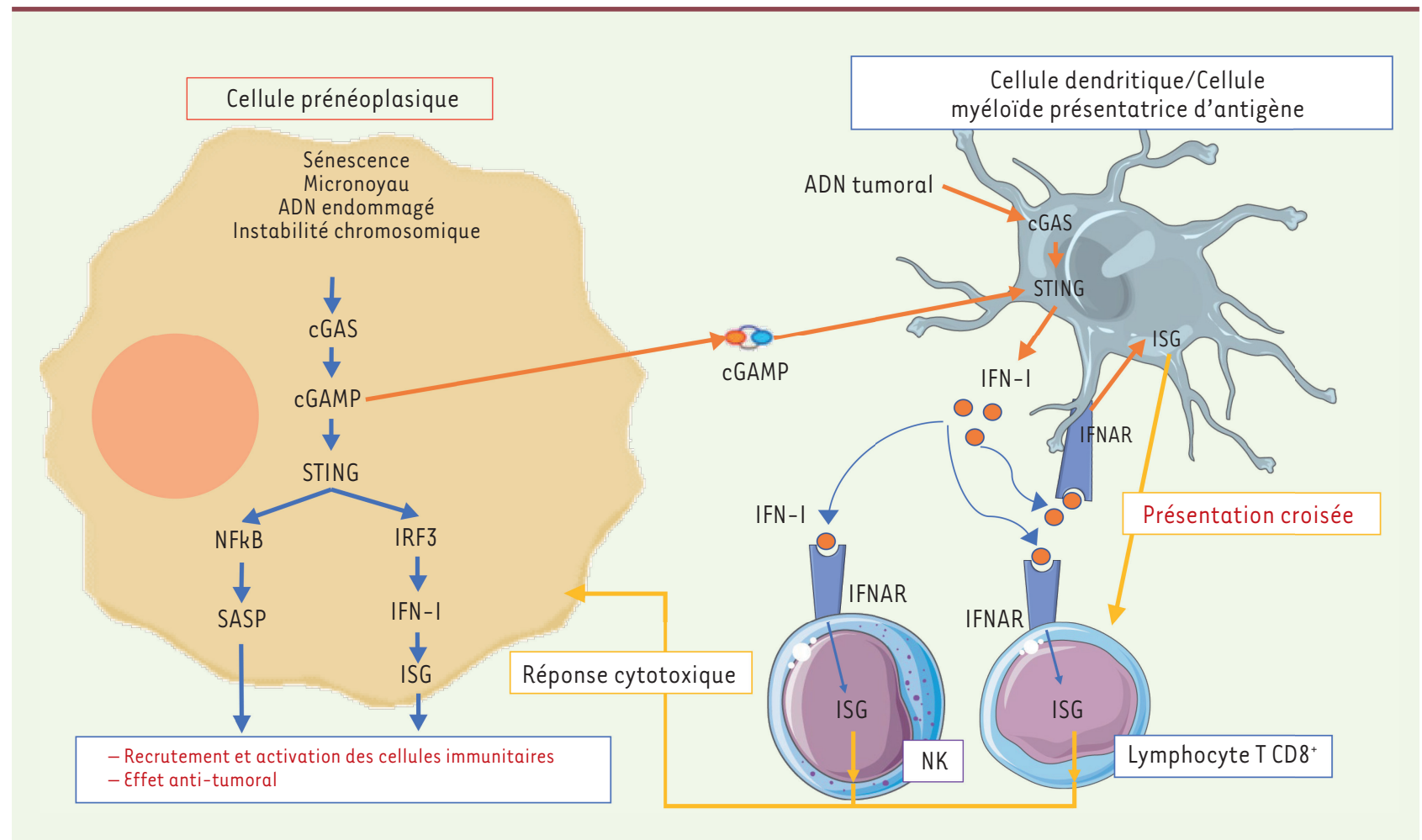

Figure 2. Rôle anti-tumoral de la voie cGAS-STING aux stades précoces de la tumorigenèse. Dans les cellules prénéoplasiques, plusieurs mécanismes (sénescence, formation de micronoyaux, instabilité chromosomique) peuvent aboutir à la présence d’ADN dans le cytoplasme qui va activer la voie cGAS-STING. Cette activation permet l'induction des gènes du SASP et des ISG (gènes induit par l'IFN-I) à l'origine de la mise en place d'une réponse anti-tumorale. L'IFN-I a la capacité d'induire l'activation des cellules dendritiques et l'amélioration de leur fonction de présentation croisée qui aboutit à une meilleure activation des lymphocytes T cytotoxiques dirigés contre les antigènes tumoraux. Le cGAMP produit dans la cellule tumorale peut diffuser dans le milieu extra-cellulaire et activer des cellules myéloïdes comme les DC, aboutissant alors à l'activation des cellules NK (natural killer) qui vont libérer le contenu de leur vésicules lytiques et détruire les cellules tumorales (adapté de [10]).

\section{La voie cGAS-STING dans l'immunothérapie}

Du fait de l'importance de la voie cGASSTING dans l'immunosurveillance des cancers, le développement de thérapies ciblant cette voie est apparu comme une évidence pour améliorer l'arsenal thérapeutique déjà existant, qui vise à activer la réponse immunitaire antitumorale. La première piste explorée a été l'utilisation d'analogues synthétiques de cGAMP. Ils présentent des modifications chimiques qui les rendent résistants à l'hydrolyse, augmentant ainsi l'efficacité anti-tumorale induite par l'activation de STING dans plusieurs modèles de souris porteuses de tumeurs. Dans deux modèles murins de cancer colorectal et de mélanome, l'injection intra-tumorale d'analo- gues synthétiques de cGAMP induit la production d'IFN-I via STING dans les $D C$, puis l'activation des lymphocytes $\mathrm{T} \mathrm{CD}^{+}$cytotoxiques dirigés contre les cellules tumorales [13]. L'administration de cGAMP à des souris porteuses de tumeurs potentialise également les effets thérapeutiques d'autres traitements, comme l'utilisation d'inhibiteurs de point de contrôle immunitaire ou la radiothérapie $[8,14,15]$.

Les résultats pré-cliniques prometteurs qui ont été obtenus ont accéléré le développement d'agonistes de STING utilisables chez l'homme. Deux études cliniques de phase I sont actuellement en cours. Elles utilisent deux agonistes de STING, I'ADU-S100 et le MK-1454, qui sont administrés par injection intratumorale dans des tumeurs solides, et dans des lymphomes [16, 17]. Malheureusement, les réponses cliniques semblent modestes, même lorsque ces agonistes sont utilisés en combinaison avec des inhibiteurs de point de contrôle immunitaire. Deux explications pouvant rendre compte de ces résultats décevants sont avancées: I'instabilité des agonistes synthétiques de STING, et la voie d'injection (intra-tumorale), qui limite l'effet de ces petites molécules. Des ligands plus stables de STING ont été développés, comme les petites molécules SR-717 et MSA-2 [18,19]. Chez les souris porteuses de tumeurs, l'administration orale ou par voie générale de SR-717 a induit une activité anti-tumorale qui favorise l'infiltration et l'activation des lymphocytes $\mathrm{T} \mathrm{CD}^{+}$cytotoxiques dans la tumeur, l'activation 
des cellules NK et l'augmentation de la présentation antigénique croisée par les DC [18]. MSA-2 a également montré un effet anti-tumoral dans un modèle de cancer colorectal chez la souris. II potentialise l'effet thérapeutique d'un inhibiteur de point de contrôle immunitaire, un anticorps monoclonal antiPD-1 (programmed cell death protein 1) $[19]$.

\section{Conclusion}

Des données récentes de la littérature ont permis de mettre en évidence le rôle majeur de la voie cGAS-STING dans l'établissement d'une barrière anti-tumorale aux étapes précoces de la tumorigenèse. Nous avons décrit l'activation de cette voie dans les cellules prénéoplasiques et dans les cellules immunitaires, et son importance dans la production d'IFN-I, à l'origine d'une réponse anti-tumorale in vivo dans des modèles murins pré-cliniques. Ces résultats ont été à l'origine du développement de molécules synthétiques agonistes de STING, stables. Ces molécules montrent des effets thérapeutiques prometteurs dans des modèles de souris porteuses de tumeurs, en monothérapie ou en association avec un inhibiteur de point de contrôle immunitaire. Ces nouveaux agonistes devraient permettre d'améliorer les traitements d'immunothérapie fondés sur les inhibiteurs de point de contrôle immunitaire et d'augmenter la survie des patients atteints de cancer. $\diamond$ The cGAS-STING pathway: A new "innate" weapon against cancer

\section{LIENS D'INTÉRÊT}

Les auteures déclarent n'avoir aucun lien d'intérêt concernant les données publiées dans cet article.

\section{RÉFÉRENCES}

1. Jiang $M$, Chen P, Wang L, et al. cGAS-STING, an important pathway in cancer immunotherapy. J Hematol Oncol $2020 ; 13: 81$.

2. Motwani M, Pesiridis $S$, and Fitzgerald KA. DNA sensing by the CGAS-STING pathway in health and disease. Nat Rev Gene 2019 ; 20 : 657-74

3. Mackenzie K.J, Carroll P, Martin CA, et al. cGAS surveillance of micronuclei links genome instability to innate immunity. Nature 2017 ; 548 : 461-5.

4. Harding S.M, Benci J.L, Irianto J, et al. Mitotic progression following DNA damage enables pattern recognition within micronuclei. Nature 2017 ; 548 : 466-70.

5. Dou Z, Ghosh K, Vizioli MG, et al. Cytoplasmic chromatin triggers inflammation in senescence and cancer. Nature 2017 ; 550 : 402-6.

6. Fuertes MB, Kacha AK, Kline J, et al. Host type I IFN signals are required for antitumor $\operatorname{CD} 8^{+} T$ cell responses through $\operatorname{CD} 8 \alpha^{+}$dendritic cells. J Exp Med $2011 ; 208$ : 2005-16.

7. Diamond MS, Kinder M, Matsushita H, et al. Type I interferon is selectively required by dendritic cells for immune rejection of tumors. J Exp Med 2011 ; 208 : 1989-2003.

8. Deng L, Liang H, Xu M, et al. STING-dependent cytosolic DNA sensing promotes radiation-induced type I interferon-dependent antitumor immunity in immunogenic tumors. Immunity $2014 ; 41$ : 843-52.

9. Marcus A, Mao AJ, Lensink-Vasan M, et al. Tumorderived cGAMP triggers a STING-mediated interferon response in non-tumor cells to activate the NK cell response. Immunity $2018 ; 49$ : 754-63.e4.

10. Kwon J, and Bakhoum SF. The Cytosolic DNA-sensing CGAS-STING pathway in cancer. Cancer Discov 2020 ; $10: 26-39$.

11. Konno H, Yamauchi S, Berglund A, et al. Suppression of STING signaling through epigenetic silencing and missense mutation impedes DNA damage mediated cytokine production. Oncogene $2018 ; 37: 2037-51$.

12. Wu S, Zhang $Q$, Zhang $F$, et al. HER2 recruits AKT1 to disrupt STING signalling and suppress antiviral defence and antitumour immunity. Nat Cell Biol 2019; 21 : 1027-40.

13. Corrales L, Glickman LH, McWhirter SM, et al. Direct activation of STING in the tumor microenvironment leads to potent and systemic tumor regression and immunity. Cell Rep 2015 ; 11 : 1018-30.

14. Demaria 0 , Gassart AD, Coso S, et al. STING activation of tumor endothelial cells initiates spontaneous and therapeutic antitumor immunity. Proc Natl Acad Sci USA 2015 ; 112 : 15408-13.

15. Woo SR, Fuertes MB, Corrales L, et al. STING-dependent cytosolic DNA sensing mediates innate immune recognition of immunogenic tumors. Immunity 2014 ; $41: 830-42$.

16. Harrington $\mathrm{KJ}$, Brody J, Ingham M, et al. Preliminary results of the first-in-human (FIH) study of MK-1454, an agonist of stimulator of interferon genes (STING), as monotherapy or in combination with pembrolizumab (pembro) in patients with advanced solid tumors or lymphomas. Ann Oncol $2018 ; 29$ : viii712.

17. Meric-Bernstam F, Sandhu SK, Hamid 0, et al. Phase lb study of MIW815 (ADU-S100) in combination with spartalizumab (PDR001) in patients (pts) with advanced/metastatic solid tumors or lymphomas. JCO $2019 ; 37: 2507$

18. Chin $\varepsilon N, Y_{u} C$, Vartabedian, VF, et al. Antitumor activity of a systemic STING-activating non-nucleotide cGAMP mimetic. Science 2020 ; 369 : 993-9

19. Pan BS, Perera SA, Piesvaux JA, et al. An orally available non-nucleotide STING agonist with antitumor activity. Science 2020 ; 369 : eaba6098. doi: 10.1126/ science.aba6098.

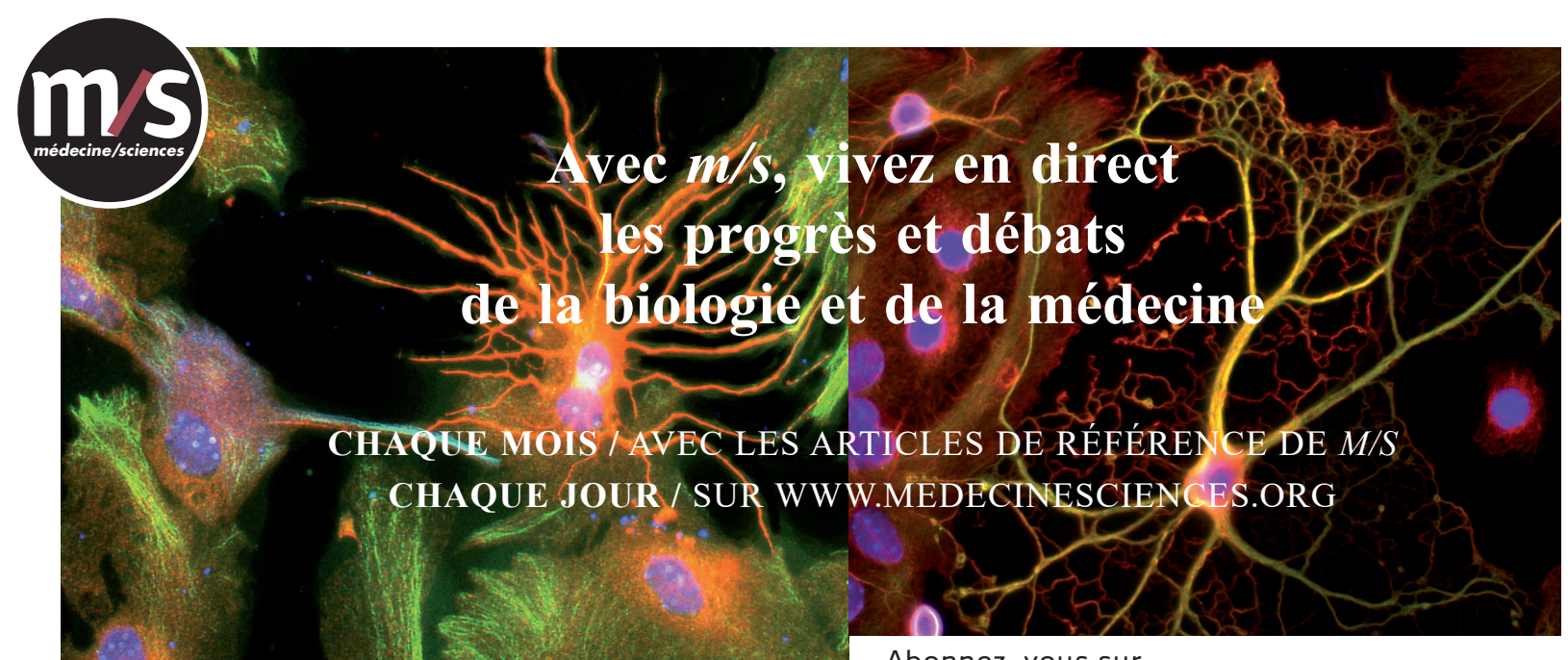

Abonnez-vous sur

www.medecinesciences.org 